\title{
Generating multivariate data from nonnormal distributions: Mihal and Barrett revisited
}

\author{
DRAKE R. BRADLEY and COURTNEY L. FLEISHER \\ Bates College, Lewiston, Maine
}

\begin{abstract}
An algorithm described by Graybill (1969) factors a population correlation matrix, $\mathbf{R}$, into an upper and lower triangular matrix, $\mathbf{T}$ and $\mathbf{T}^{\prime}$, such that $\mathbf{R}=\mathbf{T}^{\prime} \mathbf{T}$. The matrix $\mathbf{T}$ is used to generate multivariate data sets from a multinormal distribution. When this algorithm is used to generate data for nonnormal distributions, however, the sample correlations are systematically biased downward. We describe an iterative technique that removes this bias by adjusting the initial correlation matrix, $\mathbf{R}$, factored by the Graybill algorithm. The method is illustrated by simulating a multivariate study by Mihal and Barrett (1976). Large- $N$ simulations indicate that the iterative technique works: multivariate data sets generated with this approach successfully model both the univariate distributions of the individual variables and their multivariate structure (as assessed by intercorrelation and regression analyses).
\end{abstract}

A technique reviewed by Graybill (1969) can be used to generate multivariate data sets from multinormal distributions. The technique is not appropriate, however, for generating data sets from nonnormal multivariate distributions. Such distributions arise fairly often in the social sciences. Mihal and Barrett (1976), for example, reported data that clearly had nonnormal univariate distributions, as well as heterogeneous forms, across the eight variables in their study. Bradley (1993) was interested in generating multivariate data sets that would simulate the results of Mihal and Barrett's study as closely as possible. Two problems arose in the attempt to do this. First, since the distribution shapes used to model the variables had to be inferred from the descriptive statistics and scale limits reported by Mihal and Barrett (1976), the resulting distributions were approximate rather than exact. Second, when standard normal variables were correlated by the Graybill algorithm and then transformed to follow the desired nonnormal distributions, the intercorrelations were attenuated. This produced a systematic bias in the sample $r s$ (see Table 6 in Bradley, 1993). In the present report, we attempt to solve both of these problems. The actual distribution shapes for the eight variables of Mihal and Barrett's study are ascertained by examining the raw data contained in Appendix A of Mihal's doctoral dissertation (Mihal, 1974), and the attenuation in the $r$ s is eliminated by adjusting the values in the population correlation matrix to compensate for the effects of nonnormality.

The computer simulations reported in this paper were conducted on a Macintosh IIci supplied by Apple Computer and the Consortium of Liberal Arts Colleges. The author also wishes to acknowledge the support of NSF-ILI Grant USE-8852194, awarded to Bates College by the National Science foundation (G. Nigro and D. Bradley, principal investigators. Requests for reprints should be addressed to D. R. Bradley, Department of Psychology, Lewiston, ME 04240.

\section{Mihal and Barrett's (1976) Study}

Mihal and Barrett (1976) investigated the relationship between measures of cognitive style, reaction time (RT), selective attention, and susceptibility to automobile accidents. Cognitive style was assessed by the rod-and-frame test (RFT) and the embedded figures test (EFT). The four measures of RT were based on subjects' performance in an automobile simulator: initial RT, simple RT, choice RT, and complex RT. Selective attention was assessed by the number of errors made on a shadowing task. On all of these measures, high scores indicate "poorer" performance-responses that were more field dependent, slower reaction time, and poorer selective attention. The criterion variable was the number of automobile accidents that the subject had experienced in the last 5 years. Mihal and Barrett hypothesized that all of the predictor variables, except initial RT and simple RT, would correlate significantly with accidents. The results supported these predictions with the exception of choice RT, which did not correlate significantly with accidents.

To simulate Mihal and Barrett's (1976) study, we need to compute sample statistics on the raw data and use these statistics as estimates of the corresponding population parameters. The raw data are presented in the Appendix at the end of this report. Table 1 summarizes the mean, standard deviation, skew, and kurtosis for each variable, as well as the intercorrelations among the variables. Figure 1 , which shows the frequency distributions for the eight variables, confirms Bradley's (1993) earlier assertion that the variables are positively skewed and that this skew is quite extreme for the RFT, selective attention, and accident variables. (The latter have means that are only $1 S D$ or so away from the lower scale limit of $X=0$.) Comparing the values of $M$ and $S D$ in Table 1 with those reported by Mihal and Barrett (1976), we find that the values coincide, with one exception: they report $M=.52$ for the choice RT measure, and Table 1 has 
Table 1

Sample Statistics and the Correlation Matrix for Mihal and Barrett's (1976) Raw Data

\begin{tabular}{|c|c|c|c|c|c|c|c|c|}
\hline & \multicolumn{8}{|c|}{ Predictor and Criterion Variables } \\
\hline & $\begin{array}{l}\text { Rod and } \\
\text { Frame }\end{array}$ & $\begin{array}{c}\text { Embedded } \\
\text { Figures }\end{array}$ & $\begin{array}{c}\text { Initial } \\
\text { RT }\end{array}$ & $\begin{array}{l}\text { Simple } \\
\text { RT }\end{array}$ & $\begin{array}{c}\text { Choice } \\
\text { RT }\end{array}$ & $\begin{array}{c}\text { Complex } \\
\text { RT }\end{array}$ & $\begin{array}{l}\text { Selective } \\
\text { Attention }\end{array}$ & $\begin{array}{c}\text { No. of } \\
\text { Accidents }\end{array}$ \\
\hline$M$ & 4.0428 & 85.1944 & .23279 & .40380 & .51485 & .81019 & 38.23 & 1.280 \\
\hline$S D$ & 3.3569 & 45.2604 & .02196 & .03811 & .05199 & .13962 & 32.63 & 1.341 \\
\hline Skew & 1.425 & .198 & .516 & .303 & .333 & 1.791 & 1.880 & 1.035 \\
\hline Kurtosis & 4.179 & 2.191 & 3.415 & 3.708 & 2.887 & 8.275 & 6.395 & 4.022 \\
\hline \multicolumn{9}{|l|}{$r_{i j}$} \\
\hline RFT & & .528 & -.222 & -.070 & .021 & .264 & .460 & .381 \\
\hline EFT & & & -.184 & -.058 & .117 & .230 & .436 & .232 \\
\hline Initial RT & & & & .642 & .336 & .227 & -.101 & -.103 \\
\hline Simple RT & & & & & .374 & .228 & .003 & .103 \\
\hline Choice RT & & & & & & .653 & .454 & .117 \\
\hline Complex RT & & & & & & & .443 & .412 \\
\hline Selective attention & & & & & & & & .263 \\
\hline
\end{tabular}

Note- $N=75$. RFT, rod-and-frame test; EFT, embedded figures test.

$M=.51$ (rounded). Six of the correlations reported by Mihal and Barrett differ by .01 from the corresponding (rounded) values in Table 1. Two of the reported correlations, $r_{48}=.15$ and $r_{58}=.15$, compare to rounded values of $r_{48}=.10$ and $r_{58}=.12$ from Table 1 . Finally, Mihal and Barrett report $r_{68}=.27$ and $r_{78}=.40$, whereas the values in Table 1 are essentially the reverse: $r_{68}=$ .41 and $r_{78}=.26$. The cause of these discrepancies is not clear: possibly the raw data listed in Mihal's (1974) Appendix A contain one or more typographical errors. If this is the case, however, then the values of $M$ and $S D$ in Table 1 would not coincide so closely with those reported by Mihal and Barrett. With respect to $r_{68}$ and $r_{78}$, it is possible that these values were inverted when placed in the table of correlations by Mihal and Barrett or by the typesetter. Unfortunately, since Mihal is deceased and Barrett does not possess the original data (Barrett, 1992, personal communication), it is not possible to determine the source of the discrepancies in $r_{i j}$. This raises the following question: Which set of statistics-those reported by Mihal and Barrett (1976), or those presented in Table 1 -should be used for conducting simulations? Since we wish to illustrate how to generate multivariate data by using information obtained from the raw data of a study, we will take the values in Table 1 as the "correct" values, because they are computed from the data in the Appendix.

\section{The Generalized Lambda Distribution}

Given the information in Table 1, we are in a better position than Bradley (1993) to accurately simulate Mihal and Barrett's (1976) study. The main advantage comes in using the calculated values of skewness and kurtosis to select nonnormal distributions to model the variables. Doing this results in the theoretical distributions shown in Figure 2. With two exceptions noted below, these distributions have exactly the same skewness and kurtosis as do the histograms in Figure 1. The first seven plots in Figure 2 represent the probability density functions of the generalized lambda distribution (GLD). The GLD is most conveniently defined in terms of its percentile function (Ramberg, Dudewicz, Tadikamalla, \& Mykytka, 1979, p. 202):

$$
R(p)=\lambda_{1}+\frac{p^{\lambda_{3}}-(1-p)^{\lambda_{4}}}{\lambda_{2}} \quad(0 \leq p \leq 1)
$$

In this four-parameter function, $R(p)$ is the value of $X$ that corresponds to the $p$ th percentile of the GLD. To find the median of the GLD, for example, we would substitute $p=.50$ in the expression and solve for $X$. A random sample of $p$ s can be used to generate a random sample of $X s$ from the GLD, and the values of $X$ generated in this manner will follow a distribution shape that depends on the values of the lambda parameters, $\lambda_{1}-\lambda_{4}$. The central tendency $(\mu)$ and variability $(\sigma)$ of the distribution are determined by $\lambda_{1}$ and $\lambda_{2}$, respectively, and the skew $\left(\alpha_{3}\right)$ and kurtosis $\left(\alpha_{4}\right)$ of the distribution are jointly determined by $\lambda_{3}$ and $\lambda_{4}$.

To use the GLD to model empirical distributions such as those in Figure 1, it is necessary to solve for the values of $\lambda_{1}-\lambda_{4}$ that provide a GLD with the same third and fourth standardized moments about the mean ( $\alpha_{3}$ and $\alpha_{4}$ ) as the distribution being modeled. Mykytka (1978) describes methods for computing $\lambda_{1}-\lambda_{4}$ for GLDs having particular degrees of skewness $\left(\alpha_{3}\right)$ and kurtosis $\left(\alpha_{4}\right)$, and provides a FORTRAN program for doing this. Tables developed by Ramberg et al. (1979, pp. 210-214) may also be used: the tables list the values of $\lambda_{1}-\lambda_{4}$ for GLDs with $\mu=0, \sigma=1,-2.0 \leq \alpha_{3} \leq+2.0$, and $1.8 \leq \alpha_{4} \leq$ 15.8. Since the values of $\lambda_{1}-\lambda_{4}$ in these tables define "standardized" GLDs, the percentile function noted above transforms the percentiles $(p)$ to standard scores (z) sampled from the GLD. To obtain values of $X$, the values of $z$ are scaled by using the relation $X=\mu+z \sigma$, and then rounded to the appropriate number of decimal places.

To generate random samples of nonnormally distributed $X$ scores, it is necessary to obtain a sample of uniform random numbers in the interval $0 \leq U \leq 1$. This is most easily done by using a pseudorandom number 

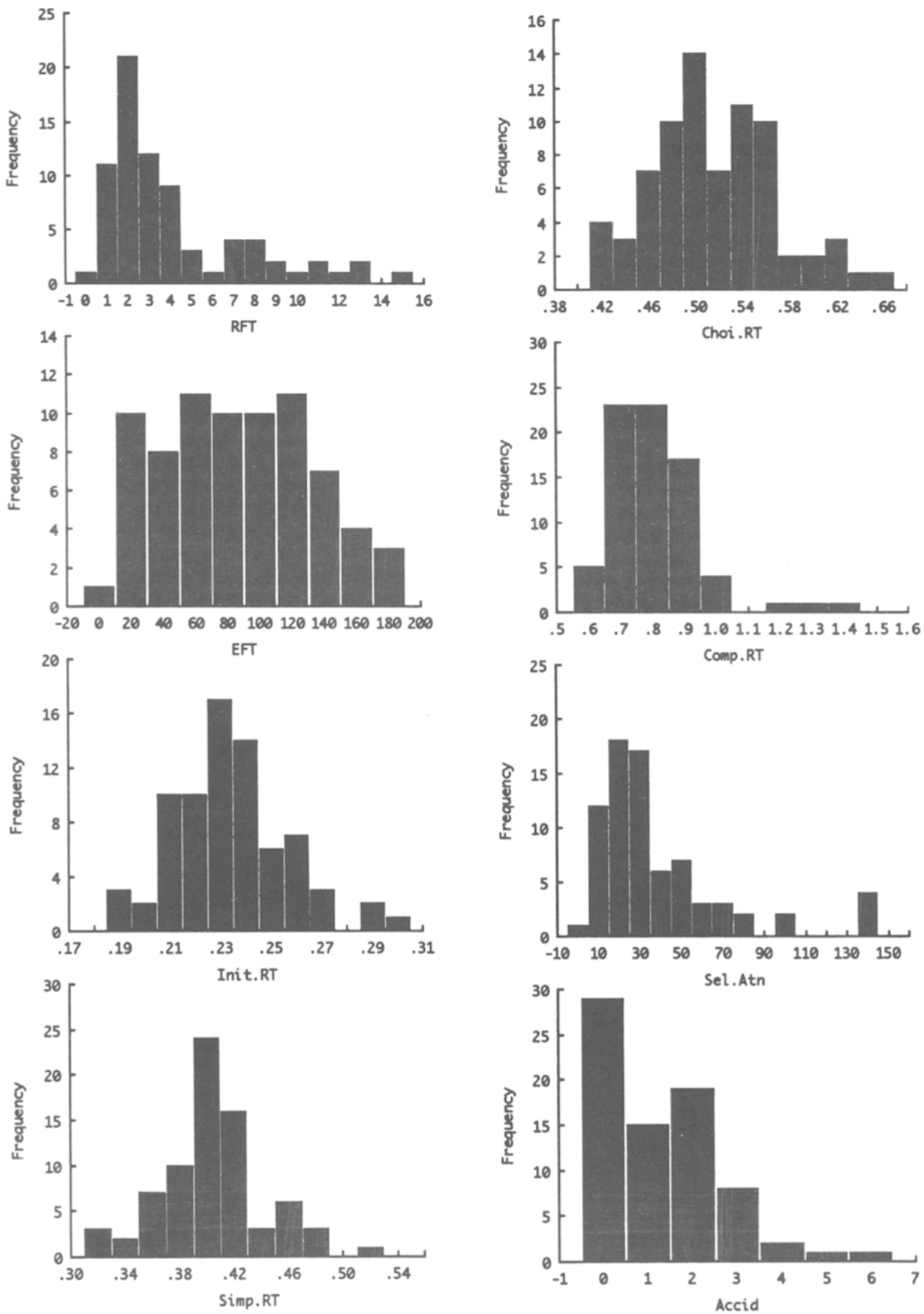

Figure 1. Frequency distributions of Mihal and Barrett's (1976) data. 

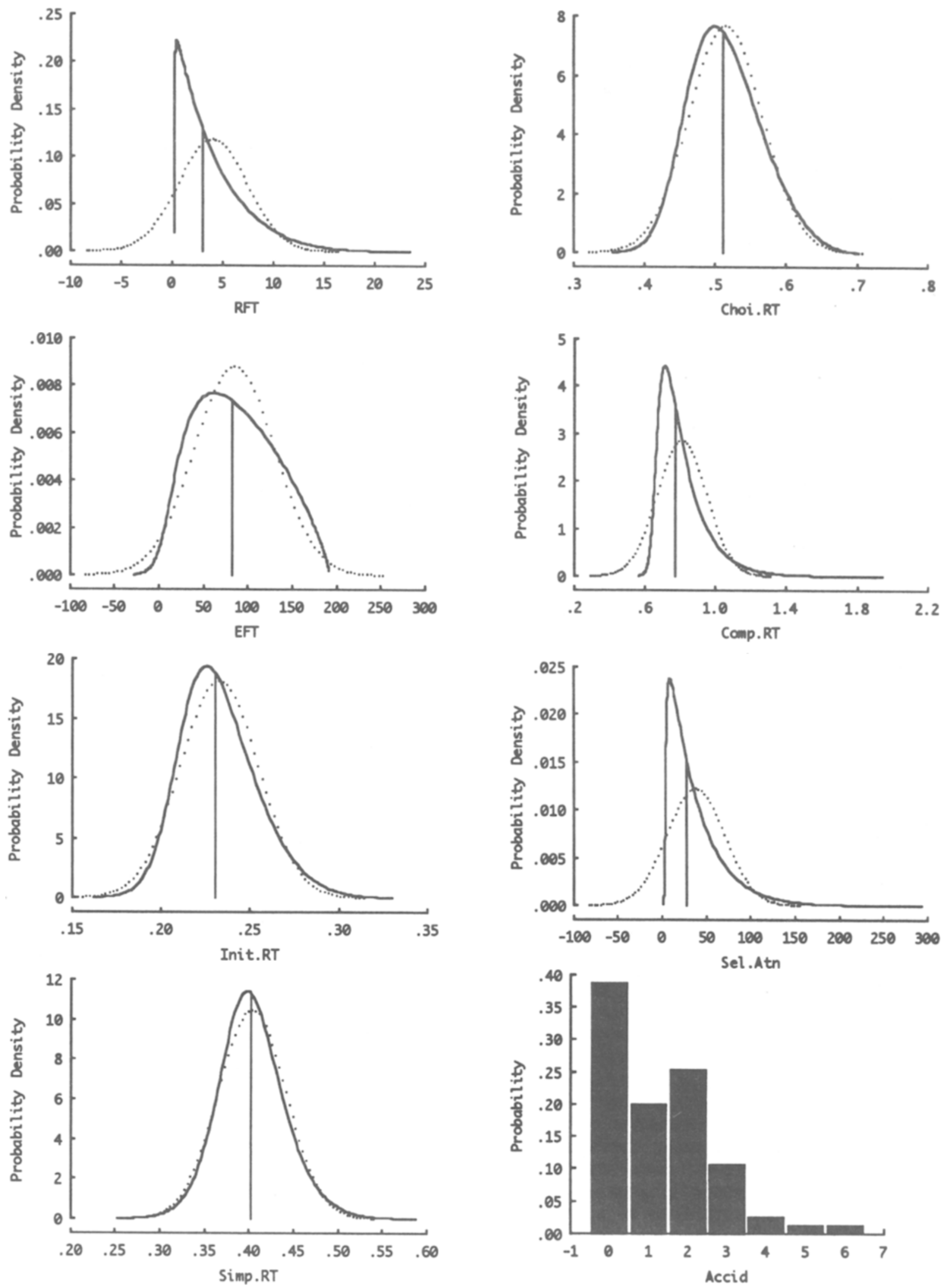

Figure 2. Theoretical distributions used to model Mihal and Barrett's (1976) data. 
generator (Lewis \& Orav, 1989, p. 65). The uniforms, interpreted as percentiles ( $p s)$, are transformed by the percentile function of the GLD to $z s$, scaled to $X$, and rounded as necessary. Since the $p$ s represent random samples from a uniform distribution, the $X$ s represent random samples from the distribution defined by the GLD. For additional information on the GLD, the reader is referred to Mykytka (1978), Ramberg and Schmeiser $(1972,1974)$, and Ramberg et al. (1979).

\section{Generating Correlated Data}

The problem becomes more complex if we wish to simulate multivariate data sets-that is, data sets in which the $k$ columns of the data matrix are intercorrelated in prescribed ways. Suppose we generate an $N \times k$ matrix $\mathbf{U}$ of uniform random numbers $\left(p_{i j} \mathrm{~s}\right)$ by making successive calls to a pseudorandom number generator. Next, the elements of this matrix are converted to standard normal deviates (Box \& Muller, 1958) in order to obtain an $N \times k$ matrix $\mathbf{Z}$. Since the $z$ scores in the $k$ columns of this matrix are derived from randomly generated uniform random numbers $(p s)$, they will be uncorrelated in the long run, although in any specific case sampling error will produce nonzero correlations among the columns of $\mathbf{Z}$. To generate multivariate data, we need to introduce systematic nonzero correlations $\left(r_{i j}\right)$ among the columns of $\mathbf{Z}$ based on the population correlations $\left(\boldsymbol{\rho}_{i j}\right)$ specified in a $k \times k$ matrix, $\mathbf{R}$. Graybill (1969, p. 298) describes an algorithm for factoring $\mathbf{R}$ into an upper and lower triangular matrix, $\mathbf{T}$ and $\mathbf{T}^{\prime}$, such that $\mathbf{R}=\mathbf{T}^{\prime} \mathbf{T}$. To correlate the $z$ scores in the columns of $\mathbf{Z}$, we simply compute the matrix $\mathbf{C}=\mathbf{Z T}$, and the values in the columns of $\mathbf{C}$ will be correlated-in the long run-as specified by $\mathbf{R}$. Because of sampling error in the matrix of randomly generated $z$ scores, the correlations among the columns of $\mathbf{C}$ will not be exactly equal to those in $\mathbf{R}$. It is precisely this feature that allows one to generate "randomized" data sets.

As long as the $z$ scores generated for the matrix $\mathbf{Z}$ are normally distributed, the long-run expected values of the correlations among the columns of $\mathbf{C}$ will be equal to the population correlations specified in $\mathbf{R}$-that is, $E\left(r_{i j}\right)=$ $\boldsymbol{\rho}_{i j}$. But what if we wish to generate correlated data sets for nonnormal distributions? If we apply different GLD transformations to the percentiles in the different columns of $\mathbf{U}$, this will convert them to the appropriate nonnormal distributions. Suppose we put these nonnormal $z$ scores in $\mathbf{Z}$, and then compute $\mathbf{C}=\mathbf{Z T}$. In the process of intercorrelating the variables by multiplying $\mathbf{Z}$ by $\mathbf{T}$, the distribution shapes are altered. Consequently, the $z$ scores in $\mathbf{C}$ will no longer conform to the desired GLDs. This suggests an alternative strategy. Suppose we convert the elements in $\mathbf{U}$ to standard normal deviates in $\mathbf{Z}$ and then compute $\mathbf{C}=\mathbf{Z T}$. Next, we convert the normally distributed (and correlated) $z$ scores in $\mathbf{C}$ back to percentiles and put these in the matrix $\mathbf{P}$. Finally, the elements in each column of $\mathbf{P}$ are transformed by using the appropriate GLD for the variable, and the results are put in G. In contrast to the previous strategy, the $z$ scores in $\mathbf{G}$ will conform to the GLD distributions because the transformation was applied following (rather than preceding) the use of the Graybill algorithm. Unfortunately, we now have the "reverse" problem: in the process of transforming the normal distributions in $\mathbf{C}$ to the desired nonnormal distributions in $\mathbf{G}$, the intercorrelations among the columns are altered. As it turns out, the correlations are reduced in value. The attenuation in the values of $r_{i j}$ relative to $\rho_{i j}$ is dependent on how heterogeneous the distribution shapes are for the two variables being correlated: the more different the shapes, the greater the attenuation.

To summarize, if we intercorrelate the $z$ scores after applying the GLD transformation, the distribution shapes are altered; if we intercorrelate the $z$ scores before applying the GLD transformation, the correlations are altered. The latter strategy is probably best: it allows one to generate multivariate data sets that in the long run have precisely the distribution shapes, central tendency, and variability specified, although the correlations will be somewhat smaller than desired. Bradley (1993, p. 159) observed that it might be possible to offset such attenuation by using values of $\rho_{i j}$ in the matrix $\mathbf{R}$ that are somewhat larger than those actually desired. As a first approximation, the increases in the values of $\rho_{i j}$ used in $\mathbf{R}$ could be equal to the amount of attenuation observed. A large$N$ simulation could then be conducted, and the values of the sample $r_{i j}$ s compared with the originally desired values of $\rho_{i j}$. The differences between the two could be used to increment or decrement the values in $\mathbf{R}$, as necessary, and the process repeated. After a few iterations, it should be possible to find an adjusted matrix for $\mathbf{R}$ that would successfully generate data sets having the desired intercorrelations.

\section{An "Exact" Simulation \\ of Mihal and Barrett's Study}

We are now in a position to attempt an exact simulation of Mihal and Barrett (1976). We regard the simulation as "exact" in the sense that every aspect of the original study that we are aware of is incorporated into the simulation. In contrast to Bradley (1993), we do not make assumptions about the distribution shapes and the scale limits of the variables. Instead, this information is obtained by analyzing the original data (see the Appendix) and by consulting Mihal's (1974) doctoral dissertation. Furthermore, an adjusted correlation matrix is employed to generate nonnormally distributed data having the same intercorrelations as the actual data.

The population parameters used to simulate Mihal and Barrett's (1976) study are summarized in Table 2. The values of $\mu, \sigma, \alpha_{3}$, and $\alpha_{4}$ are estimated by using the sample statistics presented in Table 1. An exception to this is the value of $\alpha_{4}$ for the RFT and selective attention variables. It turns out that these variables have combinations of $\alpha_{3}$ and $\alpha_{4}$ that do not correspond to a "possible" GLD (see Ramberg et al., 1979, p. 209). A decision had to be made, therefore, whether to preserve skewness or kurtosis in modeling these variables. Preserving skew is more 
Table 2

Parameters Defining the Generalized Lambda Distributions

\begin{tabular}{ccccccccc}
\hline & \multicolumn{7}{c}{ Predictor and Criterion Variables } \\
\cline { 2 - 9 } Parameters $\dagger$ & $\begin{array}{c}\text { Rod and } \\
\text { Frame }\end{array}$ & $\begin{array}{c}\text { Embedded } \\
\text { Figures }\end{array}$ & $\begin{array}{c}\text { Initial } \\
\text { RT }\end{array}$ & $\begin{array}{c}\text { Simple } \\
\text { RT }\end{array}$ & $\begin{array}{c}\text { Choice } \\
\text { RT }\end{array}$ & $\begin{array}{c}\text { Complex } \\
\text { RT }\end{array}$ & $\begin{array}{c}\text { Selective } \\
\text { Attention }\end{array}$ & $\begin{array}{c}\text { No. of } \\
\text { Accidents }\end{array}$ \\
\hline$\mu$ & 4.0428 & 85.1944 & .23279 & .40380 & .51485 & .81019 & 38.23 & 1.280 \\
$\sigma$ & 3.3569 & 45.2604 & .02196 & .03811 & .05199 & .13962 & 32.63 & 1.341 \\
$\alpha_{3}$ & 1.425 & .198 & .516 & .303 & .333 & 1.791 & 1.880 & 1.035 \\
$\alpha_{4}$ & $5.300 \ddagger$ & 2.191 & 3.415 & 3.708 & 2.887 & 8.275 & $8.200 \ddagger$ & 4.022 \\
$\lambda_{1}$ & -1.114 & -1.023 & -.4525 & -.1916 & -.4666 & -.8168 & -.9861 & -.9431 \\
$\lambda_{2}$ & .0986 & .2949 & .1447 & .08095 & .2176 & -.01633 & .01306 & .1359 \\
$\lambda_{3}$ & .0001401 & .06254 & .05526 & .03989 & .09088 & -.001654 & .0002128 & .01592 \\
$\lambda_{4}$ & .1236 & .5636 & .1336 & .05694 & .2268 & -.01477 & .01326 & .1681 \\
\hline
\end{tabular}

$\dagger \mu=E(X), \sigma=\sqrt{ } E(X-\mu)^{2}, \alpha_{3}=E(X-\mu)^{3} / \sigma^{3}, \alpha_{4}=E(X-\mu)^{4} / \sigma^{4} . \quad$ †These values were adjusted from those in Table 1, to conform to a possible GLD.

important because it determines the range of values that will be generated above and below the mean, and this aspect should be faithful to the original data. The values of $\alpha_{3}$ were therefore kept constant and the values of $\alpha_{4}$ adjusted so as to select the closest possible GLD. As a result, the GLDs used to model the EFT and selective attention data are somewhat more leptokurtic than the actual distributions.

The values of $\alpha_{3}$ and $\alpha_{4}$ for each variable were input to a FORTRAN program, LAMBDA.EXE, supplied to the authors by Mykytka (1978). The values of $\lambda_{1}-\lambda_{4}$ output by the program are shown in the lower portion of Table 2 . The probability density functions for the first seven GLDs are plotted in Figure 2, above. As it turns out, when the continuous values generated by the GLD for the accident variable are rounded to integers, a significant bias is introduced in the values of the mean and standard deviation (Bradley, 1993, p. 158). We therefore decided to use a discrete probability distribution to generate the data for this variable. The distribution is shown in the bottom right panel of Figure 2. The probabilities for $X=0$, $X=1, \ldots X=6$ were estimated from the relative frequency of occurrence of these values in the original data (see Figure 1).

If we use the correlations in Table 1 for the matrix $\mathbf{R}$, they will produce correlations smaller than desired, because of the attenuation caused by nonnormality. We need to find an "adjusted" $\mathbf{R}$ matrix which, in the long run, will generate sample correlations identical to those listed in Table 1 . To do this, an $N=100,000 \times k=8$ matrix of standard normal deviates, $\mathbf{Z}$, is generated from a known seed. The correlations in Table 1 are used to initialize the starting values for the population correlation matrix, $\mathbf{R}_{i=1}$, and this matrix is factored by the Graybill algorithm to obtain the upper triangular matrix, $\mathbf{T}_{i=1}$. The latter is used to intercorrelate the $k=8$ columns of $z$ scores: $\mathbf{C}_{i=1}=$ $\mathbf{Z T}_{i=1}$. Next, the GLD transformations in Table 2 are performed on the respective columns of $\mathbf{C}_{i=1}$ (via $\mathbf{C} \rightarrow \mathbf{P} \rightarrow \mathbf{G}$ ) in order to introduce the desired nonnormality. Finally, the resulting GLD $z$ scores are scaled to $X$ and rounded. The correlations among the columns of $X$ scores are computed, and the differences between the sample $r$ s and the values in Table 1 are determined. These differences are used to adjust the values in $\mathbf{R}_{i=1}$ upward, as necessary, thus producing an adjusted matrix, $\mathbf{R}_{i=2}$, to be employed in the next iteration. This second matrix is factored, and the $z$ scores in the matrix $\mathbf{Z}$ are now intercorrelated by computing $\mathbf{C}_{i=2}=\mathbf{Z T} \mathbf{T}_{i=\mathbf{2}}$. The $z$ scores in $\mathbf{C}_{i=2}$ are transformed by the GLDs, and scaled and rounded as before. The correlations among the columns of $X$ scores are again computed, and compared with the values in Table 1 . Any residual differences between the actual and desired correlations are used to adjust the values in $\mathbf{R}_{i=2}$ upward (or downward) to obtain the matrix for the next iteration, $\mathbf{R}_{i=\mathbf{3}}$. This process is repeated until the largest difference between the actual and desired correlations is less than .000050 . It generally requires between $n=5$ and $n=10$ iterations to find an adjusted matrix, $\mathbf{R}_{n}$, which does this.

Note that the matrix $\mathbf{Z}$ is the same for all iterations. This allows the effects of adjusting the correlations in $\mathbf{R}_{i}$ from one iteration to the next to be more easily ascertained, because changes in the sample $r_{i j}$ are not confounded by changes in sampling error, as would occur if a different (randomly sampled) matrix $\mathbf{Z}$ were employed for each iteration. A disadvantage of this approach is that the adjusted values in $\mathbf{R}_{n}$ on the final iteration reflect not only the effect of adjusting for attenuation in the $r_{i j}$ due to nonnormality, but also the effect of adjusting for any sampling error in the values of $r_{i j}$ relative to $\rho_{i j}$. Therefore, 10 different sets of iterations were conducted, each using a unique value of the seed for generating the matrix $\mathbf{Z}$. This produced 10 adjusted matrices of $\mathbf{R}$. Corresponding values were averaged across the matrices in order to obtain a final adjusted matrix for use in the Mihal and Barrett simulation.

\section{Simulating Mihal and Barrett's Study with DATASIM}

If we employ the adjusted correlation matrix in simulations of Mihal and Barrett's (1976) study, the long-run correlations among the variables should be fairly close to the desired (unadjusted) values. To see if this is the case, we conducted large- $N$ simulations with the statistical simulation program DATASIM (Bradley, 1988, 1989a, 1989b, 1991a, 1991b, 1993; Bradley, Hemstreet, \& Ziegenhagen, 1992; Bradley, Senko, \& Stewart, 1990). Fig- 
ure 3 shows the initialization of Mihal and Barrett's study as it would appear in DATASIM. For the most part, the commands used to specify the design, labels, and population parameters are self-explanatory. The DECIMAL command determines the number of places to the right of the decimal point to be retained in the simulated data. The values entered reflect those characterizing the raw data in the Appendix. The commands LOWER and UPPER specify the minimum and maximum possible values of $X$ for each variable. If we have selected the right GLDs to model the data, then off-scale values should arise only rarely, if at all. When they do, they are reassigned the value of the closest scale limit. Note that upper limits are set only for the EFT and selective attention variables: the values were determined from Mihal's (1974) description of how the variables were measured.

The LAMBDA command specifies the values of $\lambda_{1}-\lambda_{4}$ for the EFT, RFT, RT, and selective attention measures (C1-C7). The CATVAR command defines a discrete probability distribution for generating integer values of $X$. In the present case, it specifies the probabilities that the number of accidents (C8) will be $X=0, X=1, \ldots$ $X=6$. The pseudovalue " 0.38667 " listed after CATVAR indicates that the integer code " 0 " will be generated with a probability of $p=.38667$. Finally, the RHO command assigns the values of $\rho_{i j}$ to be used in the matrix $\mathbf{R}$ for generating correlated data. The values shown are the values of the adjusted correlation matrix, $\mathbf{R}$, that were obtained by averaging across the 10 iteration sets described above.

Once the initialization commands in Figure 3 have been entered, we can generate a simulated data set for Mihal and Barrett's (1976) study by entering SEED. If this command is followed by a number (e.g., SEED 39201234), then that number is used to initialize the uniform random number generator. DATASIM then generates an $N \times k$ data set based on that seed. It does this by making successive calls to the random number generator to obtain uniform random numbers, $p$, which are in turn converted to standard normal deviates (Box \& Muller, 1958). This is repeated until an $N \times k$ matrix of $z$ scores, $Z$, has been filled. This matrix is then multiplied by the matrix $\mathbf{T}$, obtained previously by factoring $\mathbf{R}$ (Graybill, 1969). The correlated $z$ scores in the resulting matrix, $\mathbf{C}$, are then converted back into percentiles, producing the matrix $\mathbf{P}$. The percentiles in the first seven columns of $\mathbf{P}$ are transformed by the appropriate GLDs, and the nonnormal $z$ scores that result are stored in the first seven columns of G. Next, the $z$ scores in columns 1-7 of $\mathbf{G}$ are scaled using the values of $\mu$ and $\sigma$ specified in the initialization (MU, SIGMA), and rounded as necessary (DECIMAL). Finally, the $X$ values in columns 1-7 of $\mathbf{G}$ are inspected to see if they are "on-scale" (LOWER, UPPER), and if not, they are assigned the value of the nearest scale limit.

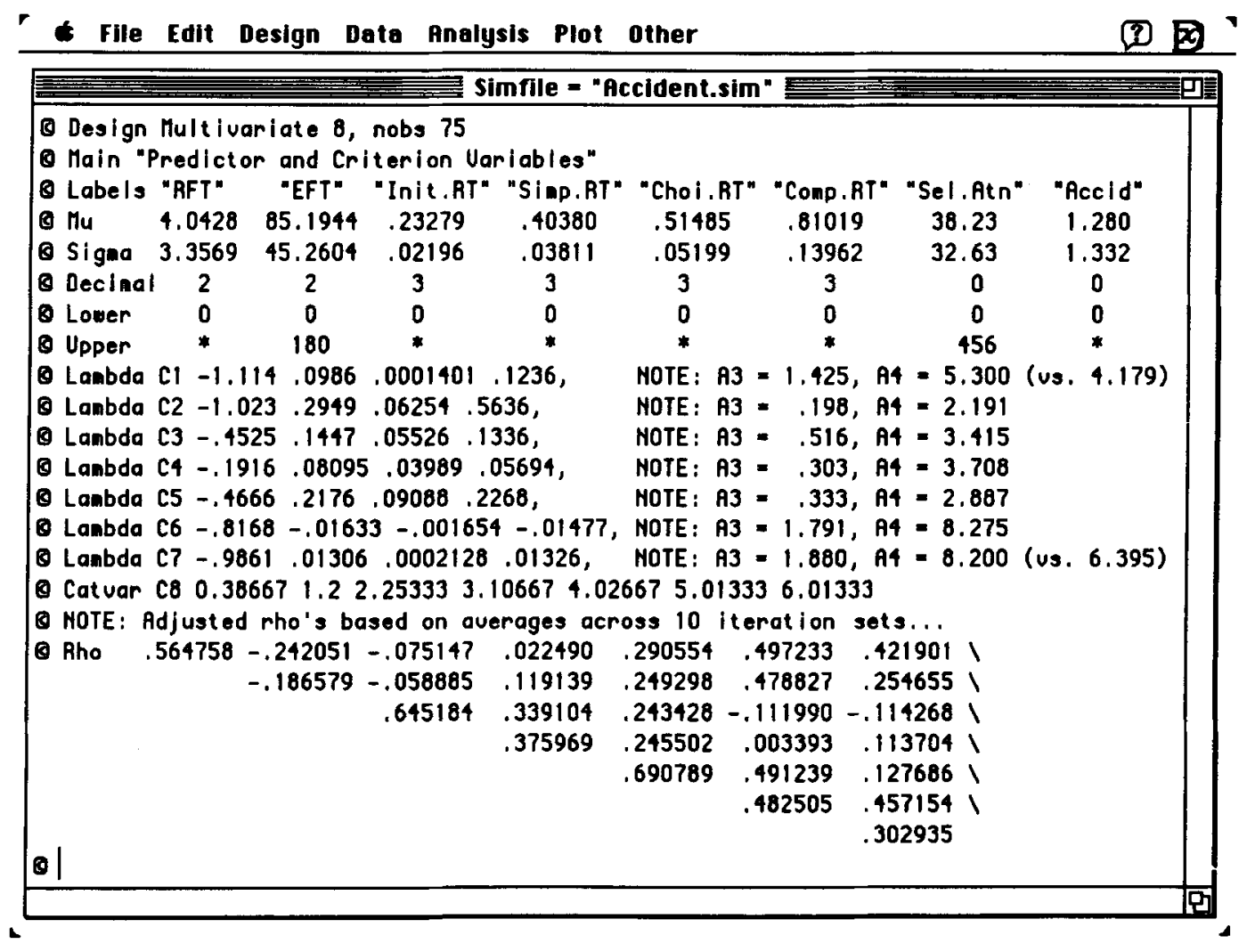

Figure 3. Initializing a simulation of Mihal and Barrett's (1976) study in DATASIM. 
What about column 8 ? Since the eighth variable (accidents) is simulated by a discrete probability distribution (CATVAR), the percentiles in column 8 of $\mathbf{P}$ are transformed to integer codes (0-6) by comparing each $p$ to the cumulative probabilities of the theoretical distribution. For example, $p=.26314$ is transformed to $X=0$, because it falls between 0 and .38667 . The integer codes resulting from these comparisons are placed in column 8 of $\mathbf{G}$. Since these values are already in their final form, they do not require scaling or rounding. And with this, the values now present in the eight columns of $\mathbf{G}$ represent a random sample of correlated $X$ values that simulate $\mathrm{Mi}$ hal and Barrett's data. The simulated data are displayed and analyzed by entering commands such as DISPLAY, STAT, CORR, MOMENTS, REGRESS C8 C1-C7, PLOT C2 C1, HIST C3, and PDF C5.

\section{Large-N Simulations of Mihal and Barrett}

We initialized a large- $N$ simulation of Mihal and Barrett's (1976) study by entering the commands in Figure 3, followed by the command NOBS 70000 . Since the statistics computed on such a large data set will have relatively little sampling error, this will allow us to more easily detect any biases arising from inaccuracies in the simulation. The simulated data set was generated by entering SEED 168752265 , and the data analyzed by entering STAT, CORR, MOMENTS, and REGRESS C8 C1-C7. One-sample statistical tests were employed to compare the sample statistics to the corresponding population pa- rameters. We will refer to this first simulation as "Sim 1" in tables of the results presented below. Keep in mind that Simulation 1 incorporates all of the relevant features characterizing Mihal and Barrett's data: nonnormal distributions, scale limits, discrete $X$ (accidents), and data sets that have "unattenuated" correlations.

In order to have a benchmark for comparison, we conducted a second simulation called "Sim 2." Simulation 2 employed normal distributions for generating all eight variables (LAMBDA C1-C8 .0000 .1974.1349 .1349), imposed no scale limits on the data, did not round data values, and used the original (unadjusted) correlation matrix from Table 1. The dotted curves in Figure 2 show the distributions used to model the variables. To facilitate comparison, a seed of 168752265 was also used to generate the Simulation 2 data set. Now, since the Graybill algorithm was developed for multinormal distributions, Simulation 2 should produce correlations consistent with the unadjusted matrix. Furthermore, since scale limits and rounding are not employed, these factors will not cause any biases in the sample statistics. Consequently, the results for Simulation 2 should conform to theoretical expectation, although the simulated data will have "impossible" values for $X$, such as negative scores for the RFT and EFT variables (see Figure 2).

The results for Simulations 1 and 2 are summarized in Tables 3-5. Table 3 compares the sample statistics obtained for the Simulation 1 and 2 data sets to the population values of $\mu, \sigma, \alpha_{3}$, and $\alpha_{4}$. One-sample $z$ tests were

Table 3

Population Versus Sample Statistics for Simulations 1 and $2(N=70,000)$

\begin{tabular}{|c|c|c|c|c|c|c|c|c|}
\hline \multirow[b]{2}{*}{ Statistic } & \multicolumn{8}{|c|}{ Predictor and Criterion Variables } \\
\hline & $\begin{array}{c}\text { Rod and } \\
\text { Frame }\end{array}$ & $\begin{array}{c}\text { Embedded } \\
\text { Figures }\end{array}$ & $\begin{array}{c}\text { Initial } \\
\text { RT }\end{array}$ & $\begin{array}{c}\text { Simple } \\
\text { RT }\end{array}$ & $\begin{array}{c}\text { Choice } \\
\text { RT }\end{array}$ & $\begin{array}{c}\text { Complex } \\
\text { RT }\end{array}$ & $\begin{array}{l}\text { Selective } \\
\text { Attention }\end{array}$ & $\begin{array}{c}\text { No. of } \\
\text { Accidents }\end{array}$ \\
\hline \multicolumn{9}{|l|}{ Mean $(\mu)$} \\
\hline Population & 4.0428 & 85.1944 & .23279 & .40380 & .51485 & .81019 & 38.23 & 1.280 \\
\hline Sim 1 & 4.0544 & 84.9279 & .23276 & .40382 & .51483 & .81010 & 38.13 & 1.275 \\
\hline $\operatorname{Sim} 2$ & 4.0533 & 84.9306 & .23276 & .40384 & .51482 & .80989 & 38.12 & 1.275 \\
\hline \multicolumn{9}{|l|}{$S D(\sigma)$} \\
\hline Population & 3.3569 & 45.2604 & .02196 & .03811 & .05199 & .13962 & 32.63 & 1.332 \\
\hline Sim 1 & 3.3666 & $44.9993 *$ & .02199 & .03807 & .05193 & .13985 & 32.62 & 1.327 \\
\hline $\operatorname{Sim} 2$ & 3.3587 & 45.1872 & .02200 & .03813 & .05196 & .14003 & 32.64 & 1.331 \\
\hline \multicolumn{9}{|l|}{ Skew $\left(\alpha_{3}\right)$} \\
\hline Pop 1 & 1.425 & .198 & .516 & .303 & .333 & 1.791 & 1.880 & 1.035 \\
\hline $\operatorname{Sim} 1$ & 1.420 & .210 & .519 & .273 & .320 & 1.797 & 1.880 & 1.035 \\
\hline Pop 2 & .000 & .000 & .000 & .000 & .000 & .000 & .000 & .000 \\
\hline Sim 2 & .006 & .004 & .004 & -.018 & -.015 & -.004 & .009 & -.001 \\
\hline \multicolumn{9}{|l|}{ Kurtosis $\left(\alpha_{4}\right)$} \\
\hline Pop 1 & 5.300 & 2.191 & 3.415 & 3.708 & 2.887 & 8.275 & 8.200 & 4.022 \\
\hline Sim 1 & 5.253 & 2.148 & 3.411 & 3.621 & 2.889 & 8.365 & 8.190 & 4.024 \\
\hline Pop 2 & 3.000 & 3.000 & 3.000 & 3.000 & 3.000 & 3.000 & 3.000 & 3.000 \\
\hline $\operatorname{Sim} 2$ & 2.986 & 2.983 & 2.993 & 2.974 & 3.017 & 2.997 & 2.992 & 3.000 \\
\hline \multicolumn{9}{|l|}{ Minimum $X$} \\
\hline Sim 1 & .25 & .00 & .155 & .212 & .333 & .541 & 0 & 0 \\
\hline Sim 2 & -8.97 & -91.98 & .148 & .247 & .304 & .256 & -90 & -4 \\
\hline \multicolumn{9}{|l|}{ Maximum $X$} \\
\hline Sim 1 & 25.53 & 180.00 & .340 & .620 & .715 & 2.459 & 397 & 6 \\
\hline Sim 2 & 17.16 & 267.55 & .319 & .555 & .728 & 1.387 & 172 & 7 \\
\hline
\end{tabular}

Note-One-sample $z$ test comparing a sample statistic to a population parameter: ${ }^{*} p<.05$. Simulation 1 employed nonnormal distributions, rounded $X$ values, lower limits of $X=0$, and an adjusted correlation matrix; Simulation 2 employed normal distributions, unrounded values, no scale limits, and an unadjusted correlation matrix. 
Table 4

Population Versus Sample rs for Simulations 1 and $2(N=70,000)$

\begin{tabular}{|c|c|c|c|}
\hline$r$ & Population & $\operatorname{Sim} 1$ & $\operatorname{Sim} 2$ \\
\hline$r_{12}$ & .528 & .529 & .528 \\
\hline$r_{13}$ & -.222 & -.218 & -.219 \\
\hline$r_{14}$ & -.070 & -.070 & -.070 \\
\hline$r_{15}$ & .021 & .021 & .022 \\
\hline$r_{16}$ & .264 & .269 & .266 \\
\hline$r_{17}$ & .460 & .457 & .458 \\
\hline$r_{18}$ & .381 & .384 & .379 \\
\hline$r_{23}$ & -.184 & -.183 & -.184 \\
\hline$r_{24}$ & -.058 & -.058 & -.059 \\
\hline$r_{25}$ & .117 & .114 & .113 \\
\hline$r_{26}$ & .230 & .230 & .229 \\
\hline$r_{27}$ & .436 & .440 & .438 \\
\hline$r_{28}$ & .232 & .235 & .232 \\
\hline$r_{34}$ & .642 & .642 & .642 \\
\hline$r_{35}$ & .336 & .339 & .339 \\
\hline$r_{36}$ & .227 & .229 & .230 \\
\hline$r_{37}$ & -.101 & -.098 & -.099 \\
\hline$r_{38}$ & -.103 & -.105 & -.104 \\
\hline$r_{45}$ & .374 & .374 & .375 \\
\hline$r_{46}$ & .228 & .227 & .227 \\
\hline$r_{47}$ & .003 & .004 & .002 \\
\hline$r_{48}$ & .103 & .101 & .101 \\
\hline$r_{56}$ & .653 & .654 & .655 \\
\hline$r_{57}$ & .454 & .454 & .452 \\
\hline$r_{58}$ & .117 & .115 & .115 \\
\hline$r_{67}$ & .443 & $.450 *$ & .446 \\
\hline$r_{68}$ & .412 & .410 & .410 \\
\hline$r_{78}$ & .263 & .267 & .264 \\
\hline
\end{tabular}

Note-One-sample $r$-to- $z$ test comparing sample $r$ to population $\rho$ : ${ }^{*} p<.05$.

used to compare the sample means and SDs to $\mu$ and $\sigma$, respectively. Only one significant result was obtained: in Simulation 1, the standard deviation of the EFT data was significantly smaller than expected $(p=.0309)$. Since 16 significance tests were conducted at $\alpha=.05$, we would expect about one Type I error to arise from chance. This result may well be that Type I error. Alternatively, it may reflect a very small bias caused by rounding offscale (negative) values of the EFT to a lower scale limit of 0 .

Two sets of population values, Pop 1 and Pop 2, are shown in Table 3 for the measures of skew and kurtosis. The Pop 1 values correspond to those of the various GLDs employed in Simulation 1, and they should be compared with the Simulation 1 sample statistics. The Pop 2 values are simply those which define a normal distribution $\left(\alpha_{3}=0\right.$ and $\left.\alpha_{4}=3\right)$, and they should be compared with the Simulation 2 sample statistics. As is evident, the skew- ness and kurtosis values obtained in both simulations correspond closely to the population values. Table 3 also shows the minimum and maximum values of $X$ that were obtained in the Simulation 1 and 2 data sets. When compared with the means, the minimum and maximum values of the Simulation 1 data clearly reflect the positive skew in the distributions of the variables. As anticipated, the normal distributions of the Simulation 2 data produce negative $X$ scores for the RFT, EFT, selective attention, and accident measures.

Table 4 shows the sample correlations for the Simulation 1 and 2 data in relation to the population values in Table 1. One-sample $r$-to- $z$ tests were used to compare the sample $r$ s to the corresponding values of $\rho$. The only significant result was the comparison of $r_{67}$ to $\rho_{67}$ for Simulation $1(p=.0242)$. Since 28 tests were conducted on the Simulation 1 results, this could well represent a Type I error. Inspection of Table 4 shows that the sample correlations for Simulation 1 are generally as close to the population values as those of the benchmark, Simulation 2. Consequently, the adjusted matrix used in Simulation 1 (see Figure 3) successfully compensates for the attenuation produced by introducing nonnormality in the data. Of course, a related question concerns the multiple regression solutions for the data: does the adjusted matrix produce data sets that have the same multiple regression solution as the original data? Table 5 provides this information. The first row shows the regression coefficients obtained when regressing the criterion variable (C8) against the predictor variables ( $\mathrm{C} 1-\mathrm{C} 7)$ for Mihal and Barrett's (1976) data (Appendix). The second row shows the population regression coefficients for the initialization of Simulation 2 in DATASIM, as output by entering REGINFO C8 C1-C7. (Simulation 2 is the relevant one to use in identifying the population values, because it employs the unadjusted correlation matrix.) The next two rows show the regression coefficients that were obtained for the regressions on the Simulation 1 and 2 data sets. Finally, note that the two columns at the far right of Table 5 list the values of $R^{2}$ and the standard error of estimate for each of these cases. Overall, there is good agreement among the respective values of the regression solution: one-sample $t$ tests comparing the Sim 1 and Sim 2 regression coefficients with the corresponding population values, or the values obtained for Mihal and Barrett's data, revealed no significant differences at $p \leq$ .05 . In contrast, Bradley (1993, pp. 157-158) reported that the Simulation 1 intercorrelations and regression statistics differed consistently from the population (and Sim-

Table 5

Population Versus Sample Regression Statistics for Simulations 1 and $2(N=70,000)$

\begin{tabular}{|c|c|c|c|c|c|c|c|c|c|c|}
\hline \multirow[b]{2}{*}{ Case } & \multicolumn{10}{|c|}{ Regression Statistics } \\
\hline & $\beta_{0}$ & $\beta_{1}$ & $\beta_{2}$ & $\beta_{3}$ & $\beta_{4}$ & $\beta_{3}$ & $\beta_{6}$ & $\beta_{\eta}$ & $R^{2}$ & $\sigma_{\text {res }}$ \\
\hline Data & .162 & .0822 & -.000298 & -15.4 & 8.93 & -6.13 & 4.80 & .00138 & .315 & 1.167 \\
\hline Population & .170 & .0816 & -.000296 & -15 & 8.87 & -6 & 4.77 & .00138 & .315 & 1.103 \\
\hline $\operatorname{Sim} 1$ & .219 & .0813 & -.000260 & -15.4 & 8.91 & -6 . & 4.73 & .00144 & .316 & 1.098 \\
\hline $\operatorname{Sim} 2$ & .253 & .0798 & -.000279 & -15.5 & 8.95 & -6.23 & 4.77 & .00147 & .314 & 1.102 \\
\hline
\end{tabular}


ulation 2) values. The absence of such differences in Tables 3,4 , and 5 shows that we have succeeded in accurately modeling both the univariate distributions and the multivariate structure of Mihal and Barrett's data.

\section{Conclusion}

Using the methods described in this paper to achieve highly accurate simulations of multivariate studies having nonnormal distributions may seem more trouble than it is worth. In fact, the iteration techniques described above were largely automated through the use of DATASIM command macros, and the large- $N$ simulations required to generate and test the iterations, while time consuming, were run overnight unattended. Consequently, researchers should not be deterred from using the approach developed here whenever it is important to precisely simulate multivariate data sets having nonnormal distributions.

\section{REFERENCES}

Box, G. E. P., \& Muller, M. E. (1958). A note on the generation of random normal deviates. Annals of Mathematical Statistics, 29, 610-611.

Bradley, D. R. (1988). DATASIM. Lewiston, ME: Desktop Press. BradLEY, D. R. (1989a). Computer simulation with DATASIM. Behavior Research Methods, Instruments, \& Computers, 21, 99-112.

BRADLEY, D. R. (1989b). A general purpose simulation program for statistics and research methods. In G. Garson \& S. Nagel (Eds.), $A d-$ vances in social science and computers (Vol. 1, pp. 145-186). Greenwich, CT: JAI Press.

BradLEY, D. R. (1991a). Anatomy of a DATASIM simulation: The
Doob and Gross horn-honking study. Behavior Research Methods, Instruments, \& Computers, 23, 190-207.

Bradley, D. R. (1991b). Datasim for the Macintosh. Lewiston, ME: Desktop Press.

BRADLEY, D. R. (1993). Multivariate simulation with DATASIM: The Mihal and Barrett study. Behavior Research Methods, Instruments, \& Computers, 25, 148-163.

Bradley, D. R., Hemstreet, R. L., \& Ziegenhagen, S. T. (1992). A simulation laboratory for statistics. Behavior Research Methods, Instruments, \& Computers, 24, 190-204.

Bradley, D. R., Senko, M. W., \& Stewart, F. A. (1990). Statistical simulation on microcomputers. Behavior Research Methods, Instruments, \& Computers, 22, 236-246.

GRAYBILL, F. A. (1969). Introduction to matrices with applications in statistics. Belmont, CA: Wadsworth.

LeWIS, P. A. W., \& ORAv, E. J. (1989). Simulation methodology for statisticians, operations analysts, and engineers (Vol. 1). Pacific Grove, CA: Wadsworth.

MiHAL, W. L. (1974). Individual differences in perceptual information processing and their relation to accident behavior. Unpublished doctoral dissertation, University of Rochester.

MiHAL, W. L., \& BARRETT, G. V. (1976). Individual differences in perceptual information processing and their relation to automobile accident involvement. Journal of Applied Psychology, 61, 229-233.

MYкYTKA, E. F. (1978). Some useful properties and methods for determining the parameters of the Ramberg-Schmeiser-Tukey distribution. Unpublished masters thesis, University of Iowa.

Ramberg, J. S., Dudewicz, E. J., Tadikamalla, P. R., \& Mykytka, E. F. (1979). A probability distribution and its uses in fitting data. Technometrics, 21, 201-214

RAMBERG, J. S., \& SCHMEISER, B. W. (1972). An approximate method for generating symmetric random variables. Communications of the $A C M, 15,987-990$.

RAMBERG, J. S., \& SCHMEISER, B. W. (1974). An approximate method for generating asymmetric random variables. Communications of the $A C M, 17,78-82$.

APPENDIX

Raw Data for Mihal and Barret's (1976) Study

\begin{tabular}{|c|c|c|c|c|c|c|c|c|}
\hline Subject & $\begin{array}{c}\text { RFT } \\
\text { (PRFT) }\end{array}$ & $\begin{array}{l}\text { EFT } \\
\text { (EFT) }\end{array}$ & $\begin{array}{l}\text { Init.RT } \\
\text { (IRT) }\end{array}$ & $\underset{\text { (SRT) }}{\text { Simp.RT }}$ & $\begin{array}{l}\text { Choi.RT } \\
\text { (CHRT) }\end{array}$ & $\begin{array}{c}\text { Comp.RT } \\
\text { (CORT) }\end{array}$ & $\begin{array}{c}\text { Sel.Atn } \\
\text { (SPT) }\end{array}$ & $\begin{array}{l}\text { Accid } \\
\text { (ACC) }\end{array}$ \\
\hline 1 & 1.00 & 45.83 & .228 & .382 & .536 & .861 & 31 & 0 \\
\hline 2 & 1.25 & 96.50 & .248 & .413 & .511 & .900 & 21 & 0 \\
\hline 3 & 1.38 & 85.83 & .297 & .463 & .592 & .989 & 48 & 0 \\
\hline 4 & .63 & 18.00 & .218 & .385 & .562 & .740 & 34 & 0 \\
\hline 5 & 1.00 & 65.00 & .221 & .358 & .505 & .847 & 27 & 0 \\
\hline 6 & 10.00 & 116.00 & .226 & .404 & .482 & .701 & 28 & 0 \\
\hline 7 & 3.88 & 98.50 & .244 & .411 & .524 & .890 & 63 & 0 \\
\hline 8 & 1.88 & 80.00 & .228 & .375 & .553 & .789 & 26 & 0 \\
\hline 9 & 1.63 & 112.33 & .243 & .412 & .577 & .922 & 16 & 0 \\
\hline 10 & 1.63 & 66.33 & .224 & .350 & .493 & .690 & 10 & 0 \\
\hline 11 & .38 & 35.83 & .256 & .451 & .455 & .671 & 8 & o \\
\hline 12 & 2.25 & 64.67 & .248 & .402 & .533 & .777 & 23 & 0 \\
\hline 13 & 3.00 & 20.33 & .225 & .411 & .558 & .775 & 24 & 0 \\
\hline 14 & 1.75 & 109.17 & .213 & .395 & .545 & .845 & 54 & 0 \\
\hline 15 & 2.56 & 161.00 & .229 & .404 & .567 & .907 & 30 & 0 \\
\hline 16 & 3.00 & 46.83 & .258 & .408 & .504 & .851 & 17 & 0 \\
\hline 17 & 1.13 & 15.17 & .211 & .404 & .416 & .705 & 7 & 0 \\
\hline 18 & 6.25 & 77.50 & .245 & .390 & .478 & .746 & 20 & 0 \\
\hline 19 & 1.13 & 52.83 & .288 & .484 & .556 & .874 & 34 & 0 \\
\hline 20 & 1.50 & 12.00 & .258 & .406 & .508 & .824 & 13 & 0 \\
\hline 21 & 8.75 & 128.67 & .245 & .403 & .457 & .644 & 29 & 0 \\
\hline 22 & 3.06 & 94.83 & .256 & .386 & .495 & .741 & 11 & 0 \\
\hline 23 & 1.63 & 29.67 & .244 & .457 & .519 & .639 & 18 & 0 \\
\hline 24 & 2.38 & 54.50 & .227 & .321 & .464 & .707 & 6 & 0 \\
\hline 25 & 3.63 & 132.33 & .232 & .402 & .485 & .701 & 15 & 0 \\
\hline 26 & 7.12 & 97.17 & .213 & .426 & .498 & .626 & 38 & 0 \\
\hline 27 & 4.75 & 71.23 & .210 & .395 & .493 & .675 & 25 & 0 \\
\hline
\end{tabular}


APPENDIX (Continued)

\begin{tabular}{|c|c|c|c|c|c|c|c|c|}
\hline Subject & $\begin{array}{c}\text { RFT } \\
\text { (PRFT) }\end{array}$ & $\underset{\text { (EFT) }}{\text { EFT }}$ & $\begin{array}{l}\text { Init.RT } \\
\text { (IRT) }\end{array}$ & $\underset{\text { (SRT) }}{\text { Simp.RT }}$ & $\begin{array}{l}\text { Choi.RT } \\
\text { (CHRT) }\end{array}$ & $\begin{array}{c}\text { Comp.RT } \\
\text { (CORT) }\end{array}$ & $\begin{array}{c}\text { Sel.Atn } \\
\text { (SPT) }\end{array}$ & $\begin{array}{l}\text { Accid } \\
\text { (ACC) }\end{array}$ \\
\hline $\begin{array}{l}28 \\
29 \\
30 \\
31 \\
32 \\
33 \\
34 \\
35 \\
36 \\
37 \\
38 \\
39 \\
40 \\
41 \\
42 \\
43 \\
44 \\
45 \\
46 \\
47 \\
48 \\
49 \\
50 \\
51 \\
52 \\
53 \\
54 \\
55 \\
56 \\
57 \\
58 \\
59 \\
60 \\
61 \\
62 \\
63 \\
64 \\
65 \\
66 \\
67 \\
68 \\
69 \\
70 \\
71 \\
72 \\
73 \\
74 \\
75\end{array}$ & $\begin{array}{r}1.75 \\
2.25 \\
7.88 \\
2.75 \\
4.00 \\
3.50 \\
2.75 \\
1.88 \\
2.38 \\
1.00 \\
12.75 \\
2.13 \\
7.75 \\
2.00 \\
1.38 \\
2.50 \\
4.38 \\
2.25 \\
6.75 \\
3.50 \\
2.50 \\
3.75 \\
1.63 \\
3.19 \\
1.38 \\
3.25 \\
1.63 \\
3.88 \\
4.63 \\
2.50 \\
2.25 \\
1.38 \\
7.00 \\
8.38 \\
3.63 \\
12.75 \\
4.50 \\
1.63 \\
10.88 \\
1.63 \\
2.88 \\
9.25 \\
15.00 \\
10.88 \\
11.50 \\
7.00 \\
2.00 \\
8.25\end{array}$ & $\begin{array}{r}9.33 \\
16.67 \\
139.17 \\
113.00 \\
180.00 \\
21.17 \\
47.50 \\
57.67 \\
76.33 \\
159.00 \\
131.67 \\
50.17 \\
111.00 \\
79.50 \\
51.33 \\
47.60 \\
119.23 \\
62.33 \\
131.50 \\
132.33 \\
40.50 \\
180.00 \\
98.67 \\
67.33 \\
22.17 \\
160.33 \\
106.33 \\
82.67 \\
119.50 \\
55.67 \\
99.00 \\
73.00 \\
125.17 \\
144.50 \\
74.17 \\
112.23 \\
28.30 \\
38.00 \\
103.83 \\
70.33 \\
15.50 \\
135.00 \\
114.17 \\
150.00 \\
180.00 \\
93.33 \\
49.33 \\
126.00\end{array}$ & $\begin{array}{l}.190 \\
.241 \\
.218 \\
.215 \\
.216 \\
.197 \\
.272 \\
.258 \\
.234 \\
.237 \\
.210 \\
.237 \\
.255 \\
.225 \\
.227 \\
.205 \\
.208 \\
.215 \\
.250 \\
.228 \\
.237 \\
.229 \\
.217 \\
.238 \\
.242 \\
.187 \\
.253 \\
.211 \\
.269 \\
.257 \\
.227 \\
.269 \\
.240 \\
.234 \\
.238 \\
.228 \\
.227 \\
.220 \\
.211 \\
.204 \\
.285 \\
.194 \\
.225 \\
.219 \\
.210 \\
.237 \\
.239 \\
.239\end{array}$ & $\begin{array}{l}.328 \\
.392 \\
.401 \\
.428 \\
.402 \\
.350 \\
.429 \\
.417 \\
.415 \\
.394 \\
.358 \\
.394 \\
.427 \\
.406 \\
.399 \\
.412 \\
.370 \\
.387 \\
.420 \\
.424 \\
.466 \\
.401 \\
.355 \\
.361 \\
.411 \\
.330 \\
.524 \\
.328 \\
.470 \\
.481 \\
.381 \\
.408 \\
.431 \\
.423 \\
.398 \\
.464 \\
.401 \\
.396 \\
.405 \\
.386 \\
.442 \\
.351 \\
.388 \\
.345 \\
.381 \\
.413 \\
.454 \\
.440\end{array}$ & $\begin{array}{l}.422 \\
.525 \\
.494 \\
.627 \\
.551 \\
.437 \\
.562 \\
.630 \\
.484 \\
.466 \\
.427 \\
.541 \\
.521 \\
.443 \\
.483 \\
.475 \\
.530 \\
.590 \\
.545 \\
.477 \\
.479 \\
.485 \\
.435 \\
.533 \\
.549 \\
.472 \\
.621 \\
.496 \\
.522 \\
.569 \\
.513 \\
.456 \\
.530 \\
.499 \\
.650 \\
.546 \\
.465 \\
.464 \\
.499 \\
.414 \\
.501 \\
.503 \\
.553 \\
.551 \\
.531 \\
.571 \\
.498 \\
.613\end{array}$ & $\begin{array}{r}.613 \\
.790 \\
.808 \\
.840 \\
.909 \\
.738 \\
.924 \\
.931 \\
.787 \\
.669 \\
.671 \\
.797 \\
.814 \\
.662 \\
.814 \\
.748 \\
.897 \\
.852 \\
.895 \\
.675 \\
.688 \\
.713 \\
.632 \\
.794 \\
.790 \\
.662 \\
1.167 \\
.833 \\
.754 \\
.781 \\
.822 \\
.685 \\
.727 \\
.904 \\
.813 \\
.962 \\
.792 \\
.786 \\
.785 \\
.705 \\
.928 \\
.853 \\
.956 \\
1.280 \\
.923 \\
.991 \\
.734 \\
1.433\end{array}$ & $\begin{array}{r}18 \\
29 \\
81 \\
70 \\
65 \\
23 \\
23 \\
53 \\
17 \\
36 \\
39 \\
9 \\
78 \\
6 \\
21 \\
11 \\
143 \\
55 \\
99 \\
31 \\
16 \\
96 \\
17 \\
26 \\
22 \\
11 \\
72 \\
31 \\
33 \\
43 \\
19 \\
5 \\
32 \\
43 \\
143 \\
41 \\
52 \\
25 \\
48 \\
14 \\
2 \\
52 \\
143 \\
143 \\
55 \\
24 \\
31 \\
45\end{array}$ & $\begin{array}{l}0 \\
0 \\
1 \\
1 \\
1 \\
1 \\
1 \\
1 \\
1 \\
1 \\
1 \\
1 \\
1 \\
1 \\
1 \\
1 \\
1 \\
2 \\
2 \\
2 \\
2 \\
2 \\
2 \\
2 \\
2 \\
2 \\
2 \\
2 \\
2 \\
2 \\
2 \\
2 \\
2 \\
2 \\
2 \\
2 \\
3 \\
3 \\
3 \\
3 \\
3 \\
3 \\
3 \\
3 \\
4 \\
4 \\
5 \\
6\end{array}$ \\
\hline
\end{tabular}

Note-RFT, rod-and-frame test; EFT, embedded figures test; Init.RT, initial RT; Simp.RT, simple RT; Choi.RT, choice RT; Comp.RT, complex RT; Sel.Atn, selective attention; Accid, number of accidents that the subject had experienced in the last 5 years. Values, as well as column labels in parentheses, are from Individual Differences in Perceptual Information Processing and Their Relation to Accident Behavior (Appendix A, pp. 92-93), by W. L. Mihal, 1974, unpublished doctoral dissertation. Copyright 1974 by the University of Rochester. 